\title{
SPIHT Compression Technique for DICOM Images using Wavelets
}

\author{
B. Janardhana Rao ${ }^{1}$, O. Venkata Krishna ${ }^{2}$ \\ ${ }^{1}$ Department of ECE, CVR College of Engineering College, Hyderabad, India \\ Email: janardhan.bitra@gmail.com \\ ${ }^{2}$ Department of EIE, CVR College of Engineering Hyderabad, India. \\ Email: venkatakrishna.odugu@gmail.com
}

\begin{abstract}
The image viewers do not consider specific requirements and scalability for the efficient encoding and decoding for easy transmission of images in many applications. This paper presents highly scalable Set Partitioning in Hierarchical Trees (SPIHT) algorithm for Digital Imaging and Communications in Medicine(DICOM) images. This algorithm is implemented using scalable line based Discrete Wavelet Transform(DWT) encoder and decoder. The performance metrics Mean Square Error (MSE) and Peak Signal to Noise Ratio(PSNR) are considered for achieving the high quality and scalable lossless image compression. This algorithm is also compatible with many heterogeneous networks.
\end{abstract}

Index terms-MSE, PSNR, DWT, Image compression, SPIHT, DICOM images

\section{Introduction}

The medical applications requires some standards for the memory storage purpose and for easy transmission [1]. The DICOM standard was developed to provide storage and some methods for easy transmission. The DICOM format has a header and a field in this header. The header always contains information about the patient, the image and imaging modality. The header field contains the 'Transfer Syntax Unique Identification'. This identification gives the type of Image compression technique which is used Compress the image data. The main disadvantage of the DICOM image is requires higher band width for transmission because usually the DICOM pictures and images are in uncompressed format and occupies more storage space. Presently, DICOM standard supports to different compression methods like lossless JPEG, lossy JPEG, JPEG-LS (lossless and near lossless), JPEG-2000 and run-length coding (RLE) [2]. In this paper, a progressive transmission coder, namely, set partitioning in hierarchical trees (SPIHT) method used to compress the DICOM images.

The aim of the work is to design an application for the easy accessing of DICOM images in any networks like UMTS, GPRS heterogeneous networks and so on. These DICOM images are compressed by systematically using of DWT and SPHIT techniques. The wavelet-based set partitioning in hierarchical trees (SPIHT) image coding algorithm generates a continuously scalable bit stream. This means that the images at various bit-rates and quality can be produced from single encoded bi stream, without any drop in compression. When a target rate or reconstruction quality has been reached then decoder simply stops decoding.

\section{Discrete Wavelet Transform}

The most important part which identifies many signals is the low-frequency content and the high-frequency content imparts details to the signal. The details and approximations are obtained after filtering in wavelet analysis. The details are high frequency components of the signal and low-scale whereas the approximations are the high-scale and low frequency components of the signal. When the original signal passes through two complementary filters it emerges two signals and it produce doubling of samples, then down-sampling is used to prevent this problem. The DWT coefficients re produced by the process on the right which includes down sampling. The decomposition is taken place on a signal, which is broken into many low resolution components. This entire successive approximated process is called wavelet decomposition is shown in figure 1 .

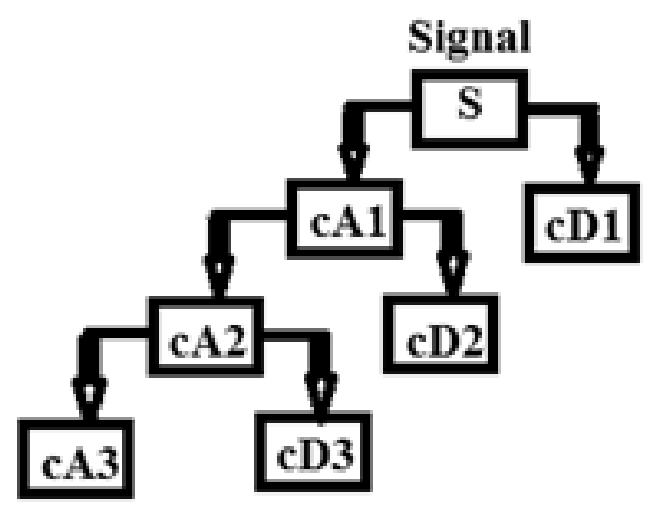

Figure 1: Decomposition in multi level

\section{A) Wavelet Reconstruction}

The Inverse Discrete Wavelet Transform (IDWT) is used for achieving the reconstruction of the image. The values are first up-sampled and then passed to the filters. The basis of the wavelet analysis is filtering and downsampling, whereas up-sampling and filtering are the basis of the wavelet reconstruction process. The Up-sampling process means simply inserting the zeros between adjacent 
samples then length of the signal component is increases. The original signal after up-sampling can be reconstructed from the coefficients of the approximations and details. Finally an approximated and detaild reconstructed signal with same length of the original signal is produced. These reconstructed details and approximations are known to be true constituents of the original signal. The down-sampling process is used for producing the details and approximations which are only half the length of the original signal. So, they cannot be directly combined to reproduce the signal [4]. Therefore, the approximations and the details are required to reconstruct before combining them. The reconstructed signal is represented as in figure 2 .

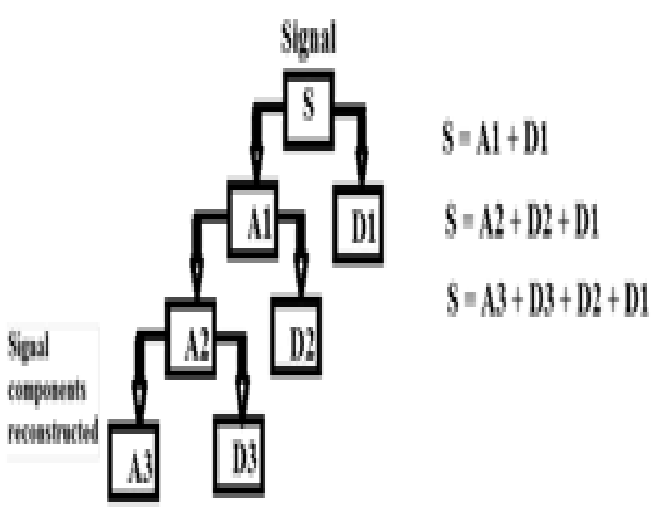

Figure 2: Signal components after reconstruction

The two-dimensional (2-D) wavelet transform can be extended from 1-D wavelet transform using separate wavelet filters. The 2-D wavelet transform of any signal can be obtained by using 1-D wavelet transform only. First by applying a 1-D wavelet transform to all the rows of the given signal, then same 1-D transform repeating on all columns of the signal [5]. The single level $(K=1) 2-D$ wavelet transform of the original signal, with corresponding notation is shown in the Figure 3.

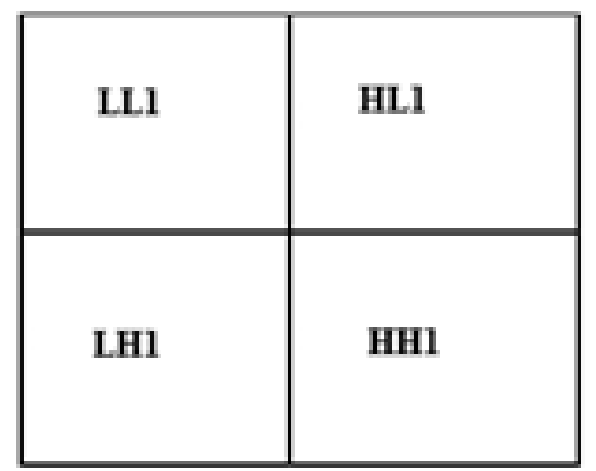

Figure 3: Single level sub band labeling scheme

The three level $(\mathrm{K}=3)$ wavelet expansion by repeating the same procedure is shown in Figure 4.

The highest level of the decomposition of the wavelet transform is represented by $\mathrm{K}$ in all discussions.

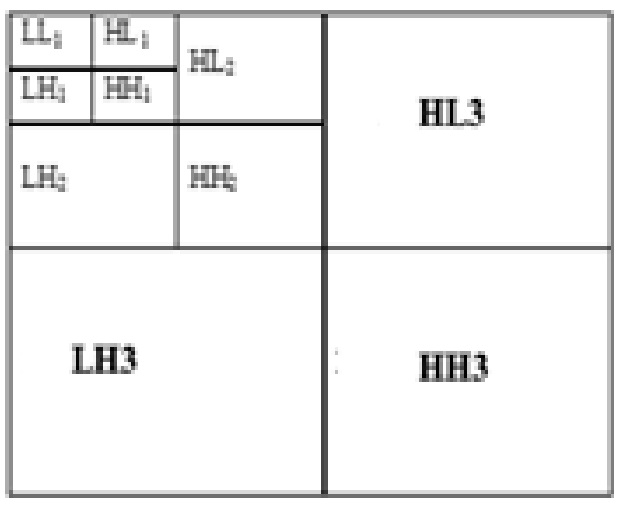

Figure 4: single level sub band labeling scheme

The 1-D version of decomposition is extended by the 2D sub band decomposition. The 1-D decomposition method takes place two times; The first decomposition is taken place in horizontal direction and the second decomposition is in the orthogonal direction. The horizontal low pass sub bands(Li) further decomposed in the vertical direction, those are LLI and LHI sub bands. In the similar way high pass sub band $(\mathrm{Hi})$ is further decomposed into $\mathrm{HLi}$ and $\mathrm{HHi}$. After this process using the same method it is possible that the LLi sub band can be further decomposed into four sub bands and this produce multiple decomposition and multiple transform levels. The first level decomposition results the four sub bands are LL1, HL1, LH1 and HH1 which is shown in the figure 4. In the second level LL1 is further decomposed in to set of four sub bands LL2, HL2, LH2 and HH2. Next the sub band LL2 is used for the third level of transform or decomposition. In any band, the low-resolution sub band is LLi and sub bands LHi, HLi, HHi are the high-pass sub bands which represent the horizontal, vertical and diagonal sub band because they represents the residual information of the original image in the horizontal, vertical and diagonal directions respectively.

The four sub bands which are low-resolution, horizontal, vertical, and diagonal produced by applying the one-dimensional transform first along the rows and then along the columns to obtain a two-dimensional wavelet transform. At each level, the wavelet transform can be reapplied to the low-resolution sub band to next de-correlate the given image. The sub band conventions, image decomposition and the defining levels used in the AWIC algorithm which is illustrated in Figure 5. The final configuration contains a less low-resolution sub band. The original image data is referred by using phrase level 0 . The original image data (level 0 ) is treated as a low-pass band when the user requests zero levels of transform and processing follows as its natural flow. 


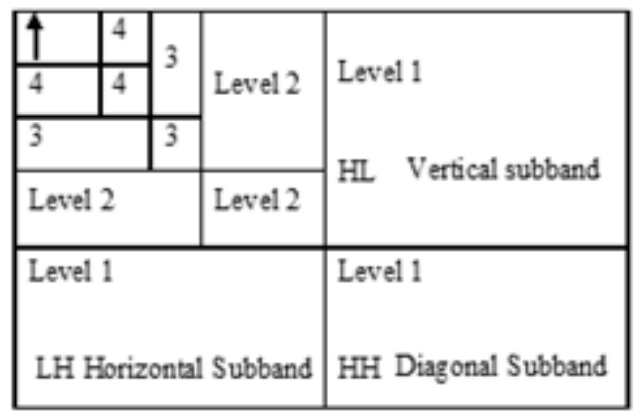

Figure 5: Image decomposition after wavelet transform

On each source image, the wavelet transformation is applied and the fusion decision map is generated. Using this map the fused wavelet coefficient map is generated from the original image wavelet coefficient. Then the fused image can be obtained from inverse wavelet transform. The fusion rules play a vital role in fusion process as explained in Figure 5.

\section{Set Partitioning In Hierarchical Trees}

The wavelet-based SPIHT image coding algorithm generates a continuously scalable bit stream. This means that the images at various bit-rates and quality can be produced from single encoded bi stream, without any drop in compression. When a target rate or reconstruction quality has been reached then decoder simply stops decoding. In the SPIHT algorithm, using hierarchical wavelet decomposition the image is first decomposed into a number of sub bands. Figure 6 shows the sub bands obtained for two-level decomposition. The spatial orientation trees are obtained by grouping the sub band coefficients, which is used for avoiding the correlation between the frequency bands. From the coefficients with highest magnitude the spatial orientation trees are coded bit plane by bit plane to the lowest pyramidal level. For further compression Arithmetic coding can also be used.

In general, the number of levels in the decomposition increases gives better compression results but the improvement becomes negligible beyond 5 levels. In practice the image dimensions limits the number of possible levels of decomposition because the wavelet decomposition can only be applied to images with even dimensions. The 5 level decomposition gives slight improved results using arithmetic coding only. To compress the binary file, a known prior information is required about the structure and properties of the image file and to exploit the abnormalities we have to assume the consistencies. The wavelet transformation produce the information about the image file then it is represented in a binary tree format. The tree contains a root and branches, where root with high probability of containing pixel magnitude level than that of the branches pixel magnitude. From this information, the algorithm is defined and named as the Set Partition in Hierarchical Tree (SPHT) algorithm.

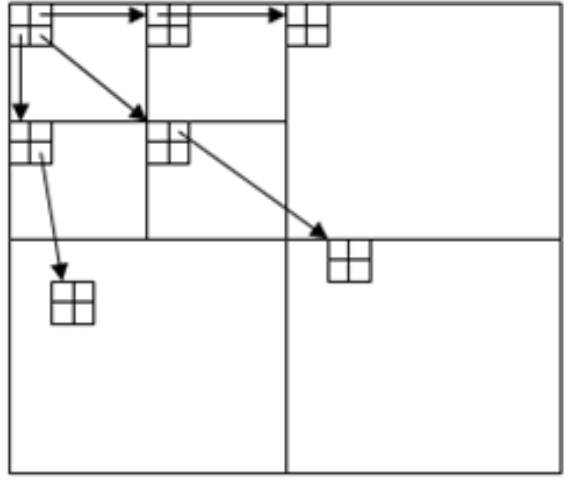

Figure 6: Spatial orientation tree by 2-level wavelet decomposition.

\section{A) Set Partitioning Algorithm}

The SPHT algorithm does not transmit the contents of the pixel values, or the pixel coordinates and the sets directly. It transmits only the decisions made in each and every step of the progression of the trees. Then these decisions define the structure of the image. These decisions gives the information of the pixel values, points, outcomes and part of the tree. Actually pixel coordinates are defined the part of the tree and pixel value defined by decision points and their outcomes. Finally, from these decisions the decoder also uses identical algorithm. This algorithm easily identifies the decisions and creates the identical sets as encode

The SPIHT part that gives the pixel values is the comparison of each pixel value. These pixel values designated to $2^{n} \leq c_{i, j} \leq 2^{n+1}$ with each pass of the algorithm by decreasing value of $n$. The decoder get the bit values each single value of $n$ without any passing of pixel values. Then the magnitude of the compression can be controlled. For the sufficient number of $\mathrm{n}$ value, many information loops being passed with less error and for the small $n$ value more pixel variation will be tolerated. Here $\mathrm{c}_{\mathrm{i}, \mathrm{j}} \leq 2^{\mathrm{n}}$ is a significant pixel value for given pass. Like this pixel values and coordinates are sorted by 'significant' and "insignificant " tags. By using these significant and insignificant tags all the pixels are placed into partition sets. The problem with multiple times of traversing through all pixel values would take more time and inefficient.

Therefore to overcome the above problem the SPIHT algorithm is considered. In this algorithm, tree sort simulation is takes place only traverse into the tree as much as needed on each pass. This entire work is done by wavelet transform only. This wavelet transform produces properties of the image. Which are required for the algorithm. This algorithm "tree" is constructed as having the root at the very upper left most pixel values and this again extending downwards into the image. In this tree, the each node having four off spring nodes(See figure 6). The SPIHT method is an important advance method for image compression. It is not an extension from the traditional methods.

In image coding field, the image-coding using the wavelet transform is popular method. Generally so many coding algorithms are using wavelet transforms. But, there are two only successful, one is "embedded zero tree 
wavelet coding" by Shapiro and the "set partitioning in hierarchical trees (SPIHT) " by Said and Pearlman. These two methods are very efficient and are able to reduce the block artifact than current standard JPEG image compression [6].

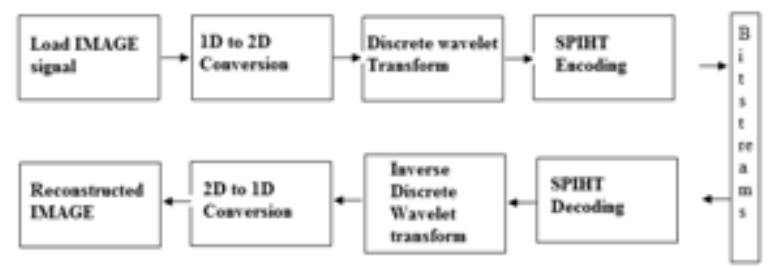

Figure 7: The block diagram for DWT-SPIHT encoding and decoding.

\section{Performance Evaluation}

The important parameter for the image compression is the compression efficiency. Which is defined and estimated by the ratio of the original image size over the compressed data size.

The next parameter is complexity of an image compression algorithm. Generally the complexity is calculated with respect to encoding and decoding process in the algorithm i.e the number of data operations required to perform both encoding and decoding processes. But, sometimes it is expressed by the number of operations only.

In general, any encoding and decoding methods some data will be lost. Hence, the distortion measurement is also an important criterion to find out how much information has been lost. In image compression, the reconstructed image after compression is not exact replica of original image. This distortion is measured by the "mean square error(MSE)". The mean square error is given by:

$$
M S E=\left(\frac{1}{N}\right) \sum_{i=0}^{N-1}\left(x_{i}^{\prime}-x_{i}\right)^{2}
$$

where $\mathrm{xi}$ is the original signal value and $x_{i}$ is the reconstructed value. $\mathrm{N}$ is the total number of pixels. Mostly the MSE is measured in decibels for the lossy codec methods that is called "Peak signal to Noise Ratio". In this paper also the PSNR objective measurement for compression methods. The PSNR is given as,

$$
P S N R=10 \log _{10} \frac{M A X^{2}}{\frac{1}{w * h} \sum_{i=1}^{w} \sum_{j=1}^{h}(o(i, j)-c(i, j))^{2}}
$$

where $\mathrm{h}$ is the height of the image and $\mathrm{w}$ is the width of the image.MAX $=255$ is maximum pixel value. $\mathrm{c}$ is compressed image date of original image 0.

\section{A) Error Metrics}

Error metrics are used to compare the different image compression techniques. Two important error metrics 1)
Mean Square-Error (MSE) and 2) Peak Signal-to-Noise Ratio (PSNR) are mostly used. The MSE gives the cumulative squared error between the original image and the compressed image and the PSNR shows the peak error[7].

The MSE and PSNR are given by,

$$
\begin{aligned}
& M S E=\frac{1}{M N} \sum_{y=1}^{N} \sum_{x=1}^{M}\left[I(x, y)-I^{\prime}(x, y)\right]^{2} \\
& P S N R=20 * \log _{10}\left(\frac{225}{\operatorname{sqrt}(M S E)}\right)
\end{aligned}
$$

\section{SIMULATION RESULTS}

The MATLAB code for Discrete wavelet transform is simulated. In this the original image is decomposed into high and low frequency signals. Next, the SPHIT algorithm encodes the resulting image of discrete wavelet transformation and sent to decompressing. The decompressing section consist of decoding and inverse discrete wavelet transform. Finally the approximated or reconstructed image to the original image is produced by the SPHIT.

The entire procedure, MATLAB code execution and the corresponding simulation results are shown in the following figures. The figure .8 represents the GUI (Graphical User Interface) window. In this window a DICOM image is browsed and the cho as in the figure .9.

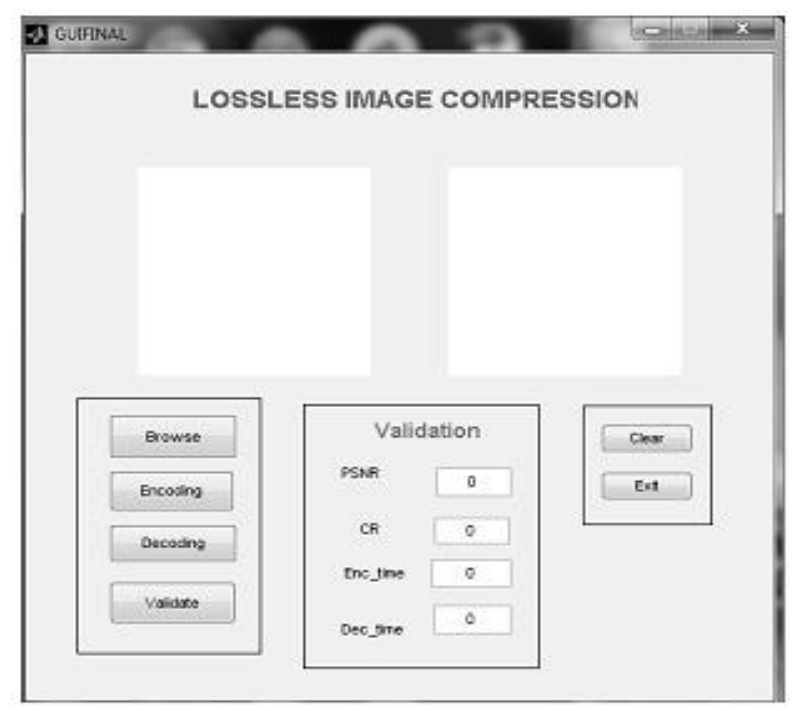

Figure 8: GUI Window after execution of code. 


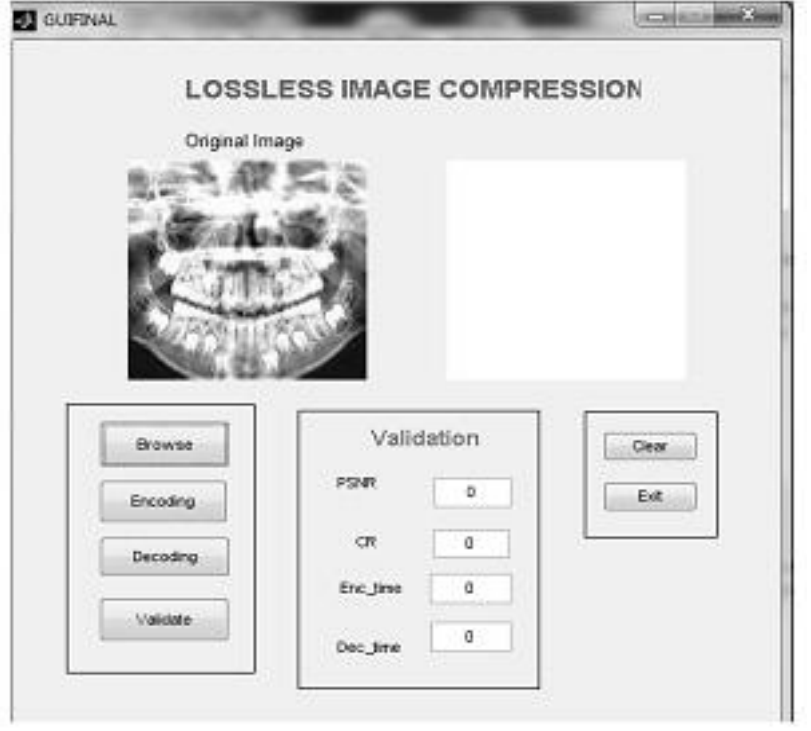

Figure 9: GUI window after browsing the image

In the GUI, by using the Encode tab the selected DICOM image is transformed into four sub-bands by Discrete Wavelet Transform. The resultant sub-bands are separated by low and high signals. Then, the transformed image is converted into bit-stream by encoding using SPIHT as shown infigure10

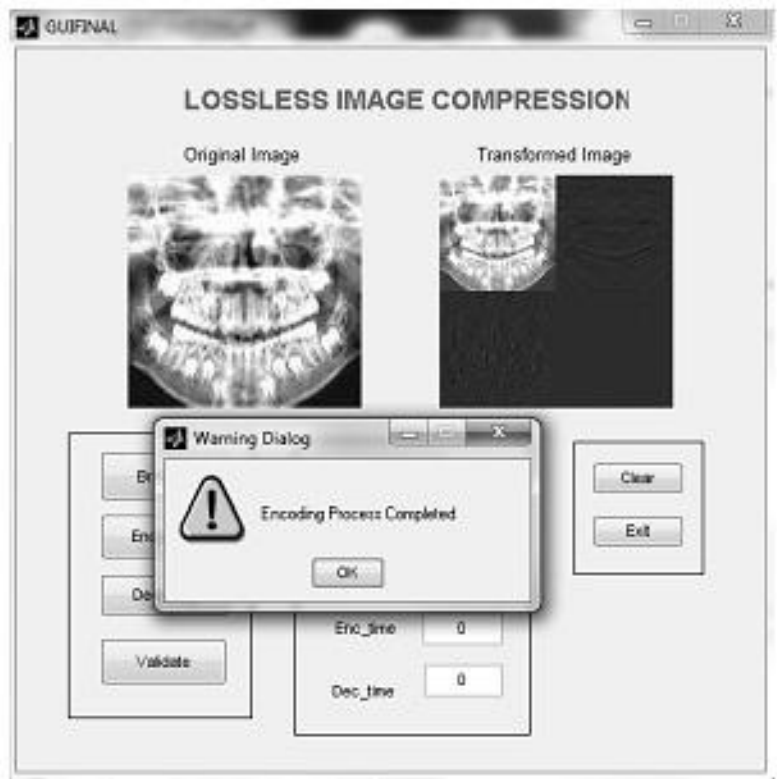

Figure 10: GUI window after encoding process

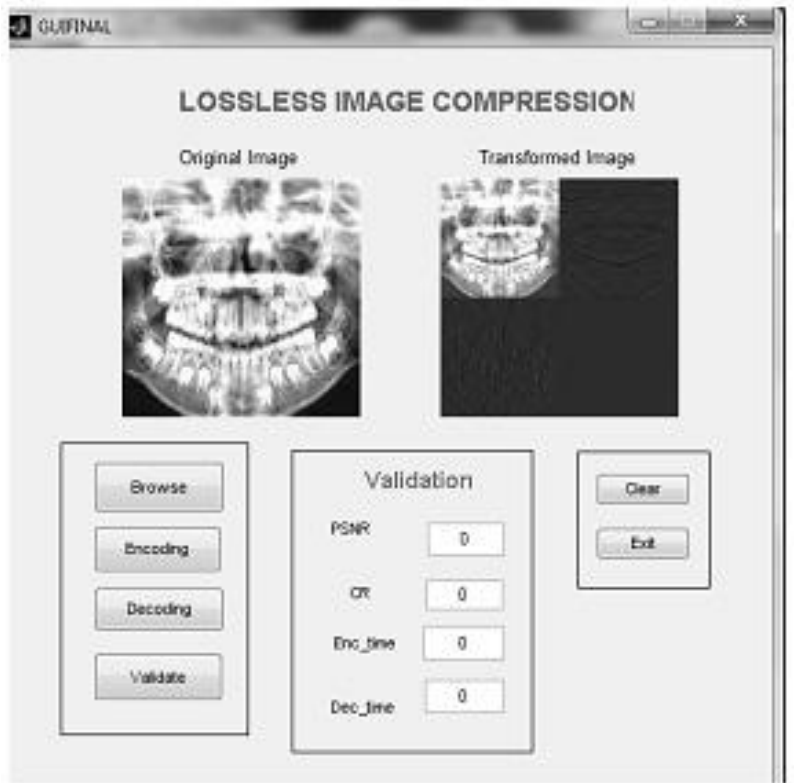

Figure 11: GUI window after encoding and ready for decoding process

At the receiving end the bit-stream is decoded and produce reconstructed image. Actually, the image is shown before decoding but the window is showing after decoding the image as shown in figure 12.

The figure. 13 represents the decompressed image or reconstructed image, which is almost the replica of the original image. This entire method provides lossless image with high compression ratio.

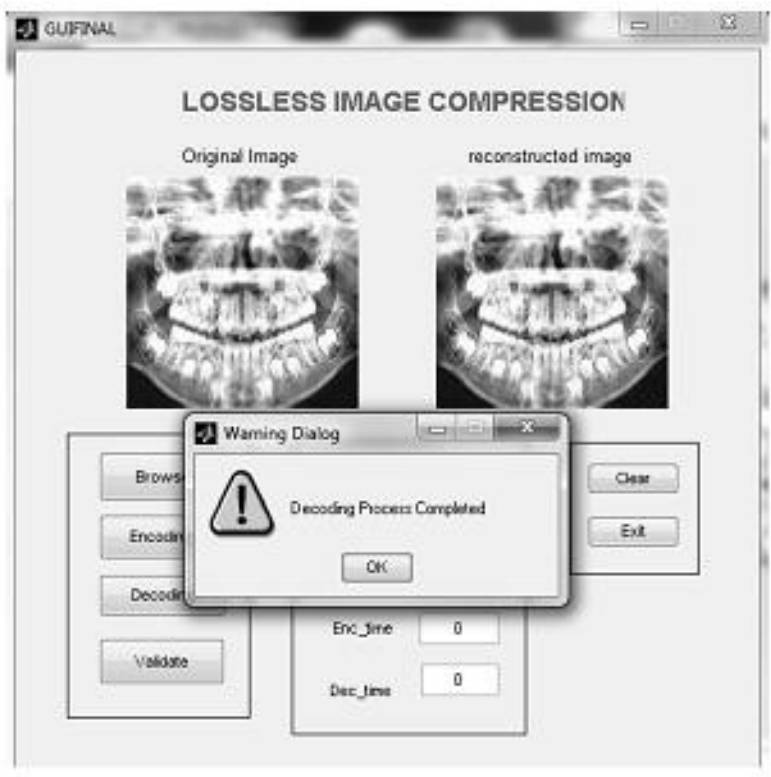

Figure 12: GUI window after decoding process 


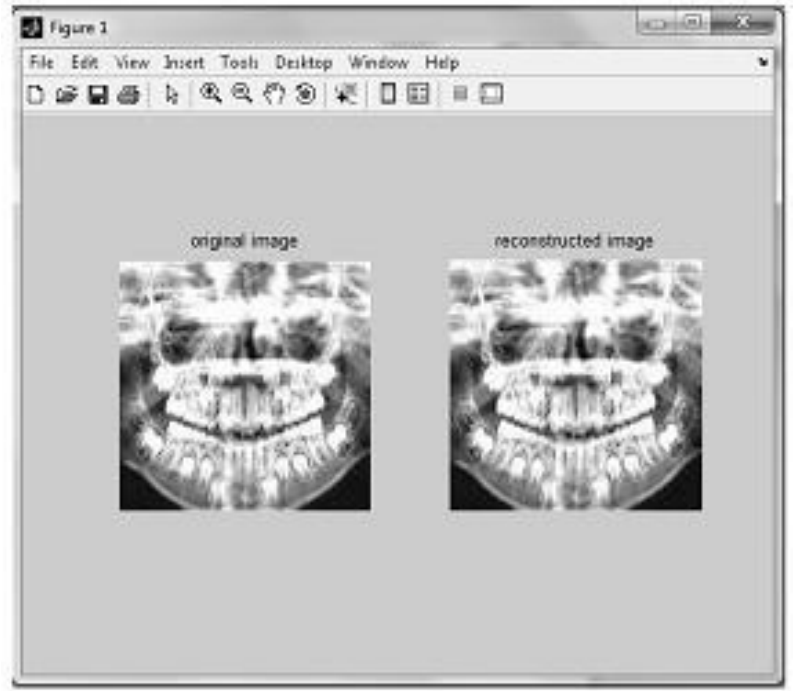

Figure 13: GUI window after reconstructed image

\section{CONCLUSIONS}

In this paper, we presented a line based Discrete wavelet transform which applied to the encoder and decoder to perform image compression. In this algorithm the encoder and decoder consists of same memory and symmetrical with respect to complexity. Based on these parameters highly optimized SPIHT coding algorithm with less memory along with line based transform is presented. Comparatively this algorithm given good performance than many image compression coding methods. In this work the computational complexity and memory is reduced by the implementation of a less memory wavelet image coder. This wavelet image coder is optimized in terms of speed and memory. The further speed improvements can be achieved by using lifting steps or lattice factorization of the wavelet kernel.

\section{REFERENCES}

[1] DICOM Standard http://medical.nema.org/dicom/2004

[2] DICOM Part 5: Data Structures and Encoding http://medical.nema.org/dicom/2004/04_05PU.PDF

[3] J. Z. Wang, "Wavelets and imaging informatics: A review of the literature" J. Biomed. Inf., vol. 34, no. 2, pp. 129-141, Apr. 2001.

[4] Myint Myint Yi, Khin Sandar Linn, and Marlar Kyaw, "Implementation of Neural Network Based Electricity Load Forecasting," World Academy of

Science, Engineering and Technology 422008.

[5] Prarthana Shrestha "MIPS Augmented with Wavelet Transform: Perfor-mance Analysis", Computer Engineering Laboratory, Faculty of Information Technology and Systems, Delft University of Technology, The Netherlands, July 2002.

[6] J. Malý, P. Rajmic, "DWT-SPIHT IMAGE CODEC IMPLEMENTATION," Department of

Telecommunications, Brno University of Technology, Brno, Czech Republic.

[7] Mallat, S.G. "A Thory of Multiresolution Signal Decomposition:The Wavelet Representation," IEEE Trans.PAMI, Vol.11, No.7, PP.674-693, July 1989. 